\title{
Assessment of postoperative complications using E-PASS and APACHE II in patients undergoing oral and maxillofacial surgery
}

Kiyohide Ishihata ${ }^{{ }^{*}}$, Yasuyuki Kakihana ${ }^{2}$, Takuya Yoshimura', Juri Murakami ${ }^{1}$, Soichiro Toyodome ${ }^{1}$, Hiroshi Hijioka', Etsuro Nozoe ${ }^{1}$ and Norifumi Nakamura ${ }^{1}$

* Correspondence: ishihata@dent. kagoshima-u.ac.jp

${ }^{1}$ Department of Oral and Maxillofacial Surgery, Field of Maxillofacial Rehabilitation, Kagoshima University Graduate School of Medical and Dental Sciences, 8-35-1 Sakuragaoka, Kagoshima 890-8544, Japan Full list of author information is available at the end of the article

\begin{abstract}
Background: The prediction of postoperative complications is important for oral and maxillofacial surgeons. We herein aimed to evaluate the efficacy of the Estimation of Physiologic Ability and Surgical Stress (E-PASS) and Acute Physiology, Age, and Chronic Health Evaluation (APACHE) II scoring systems to predict postoperative complications in patients undergoing oral and maxillofacial surgery.
\end{abstract}

Methods: Thirty patients (22 males, 8 females; mean age: $65.1 \pm 12.9$ years) who underwent major oral surgeries and stayed in the intensive care unit for postoperative management were enrolled in this study. Postoperative complications were discriminated according to the necessity of the therapeutic intervention by the Medical Department, i.e. according to the Clavien-Dingo classification. E-PASS and APACHE II scores as well as laboratory test values were compared between patients with/without postoperative complications.

Results: Postoperative complications were developed in seven patients. The comprehensive risk score (CRS: $1.13 \pm 0.24)$ and APACHE II score (13.0 \pm 2.58$)$ were significantly higher in patients with postoperative complications than in those without ones ( $p<0.01, p<0.05$, respectively). The CRS showed an appropriate discriminatory power for predicting postoperative complications (area under the curve: 0. 814). Furthermore, a correlation was detected between APACHE $\|$ scores and postoperative data until C-reactive protein levels decreased to $<1.0 \mathrm{mg} / \mathrm{L}(r=0$. $43, p<0.05)$.

Conclusion: The E-PASS and APACHE II scoring systems were both shown to be useful to predict postoperative complications after oral and maxillofacial surgery.

Keywords: Oral and maxillofacial surgery, Estimation of Physiologic Ability and Surgical Stress (E-PASS), Acute Physiology, Age, and Chronic Health Evaluation (APACHE) II, Postoperative complications

\section{Background}

Risk management for postoperative complications is important for patients and surgeons. Surgical interventions lead to the production of proinflammatory cytokines, which may result in the development of systemic inflammatory response syndrome (SIRS) [1, 2]. These biological responses are regarded as beneficial because they increase immune functions and promote tissue repair; however, if the reserve competence of a patient cannot

(c) The Author(s). 2018 Open Access This article is distributed under the terms of the Creative Commons Attribution 4.0 International License (http://creativecommons.org/licenses/by/4.0/), which permits unrestricted use, distribution, and reproduction in any medium, provided you give appropriate credit to the original author(s) and the source, provide a link to the Creative Commons license, and indicate if changes were made. The Creative Commons Public Domain Dedication waiver (http://creativecommons.org/ publicdomain/zero/1.0/) applies to the data made available in this article, unless otherwise stated. 
withstand surgical stress, homeostasis may collapse, and as a consequence, various postoperative complications may develop. Postoperative complications such as surgical site infection (SSI), aspiration pneumonia, and swallowing and breathing difficulties have a significant impact on the prognosis of patients, and as a result, increase health care costs and hospitalization [3]. Therefore, many surgeons have examined patients based on their performance statuses, biological ages, and clinical test results.

Several scoring systems have recently been developed to predict postoperative morbidity and mortality in an attempt to prevent unfavorable outcomes after general surgery; however, there have been few studies on the predictors of postoperative complications in the field of oral and maxillofacial surgery for oral surgeons. The estimation of physiologic ability and stress (E-PASS) scoring system is a useful and simple strategy to predict postoperative mortality and morbidity [2, 4]. It evaluates the physiological condition of a patient and surgical invasion and precisely reflects the general condition of a patient in a perioperative setting. Acute Physiology, Age, and Chronic Health Evaluation (APACHE) II, a severity of disease classification system that use basic physiological principles, has frequently been applied in many Intensive Care Units (ICU) to stratify prognosis of acute ill patients [5, 6].

Since the oral and maxillofacial regions with its important arteries and veins or nerves play a major role in ingestion and breathing, reliable predictors for postoperative complications are needed. However, few studies have been performed to identify predictive risk factors related to postoperative complications in patients who undergo oral and maxillofacial surgery.

Thus, we herein aimed to identify risk factors that correlate with postoperative complications in patients who underwent oral and maxillofacial surgery with relatively high surgical stress in our hospital. Furthermore, this is the first attempt to assess the utility of the E-PASS and APACHE II scoring systems in this field.

\section{Methods}

\section{Patient characteristics}

Thirty patients who were treated for oral cancer or oral benign tumors with resection and reconstruction in the Department of Oral and Maxillofacial Surgery at a university hospital in Kagoshima, and admitted to the ICU for postoperative management between 2013 and 2015 were reviewed retrospectively. An Institutional Review Board approved this retrospective observational study. Patients included 22 men and eight women with a mean age of $66.9 \pm 11.0$ years. The original disease was a malignant tumor in 29 patients and benign tumor in one patient. Radical neck dissection was performed on 21 patients, supraomohyoid neck dissection on six patients, and upper neck dissection on one patient. Regarding reconstruction, a pectoralis major myocutaneous flap was used in nine patients, forearm flap in 9, latissimus dorsi flap in 6, and delto-pectoral flap in three patients. In all patients, the same operator team at the same hospital performed tumor resection, neck resection, and flap reconstruction. Duration of follow-up period was 2.5 years.

Postoperative complications were discriminated according to the necessity of the therapeutic intervention by the Medical Department, i.e. according to the Clavien Dingo classification [7]. In this classification, surgical complications were categorized from grade 1 to 5 based on the invasiveness of the treatment required. Grade 1 requires 
no treatment; grade 2 needs medical therapy; grade 3a requires surgical, endoscopic, or radiological intervention, but no general anesthesia; grade $3 \mathrm{~b}$ requires general anesthesia; grade 4 represents life-threatening complications that require intensive care; and grade 5 represents complications leading to patient death. In this study, the grade 2 or above patients were determined to have postoperative complications. Thus, patients were classified into two groups based on whether they developed postoperative complications.

\section{Scoring systems and laboratory test values E-PASS and APACHE II scoring systems}

We investigated E-PASS and APACHE II score variables and evaluated postoperative courses. The development of E-PASS has already been described in detail [2]. Briefly, the original E-PASS consisted preoperative risk score (PRS), which reflects reserve capacity, surgical stress score (SSS), which reflects surgical stress, and the comprehensive risk score (CRS), in which PRS and SSS are combined. APACHE II uses a point score based on 12 routine physiological measurements (ranges from 0 to 60), age score (from 0 to 6), and previous health status score (from 0 to 5 ) to provide a general measure of the severity of disease [5] (Table 1).

\section{Laboratory test values}

On the preoperative day, a physical examination of cardiac and respiratory functions was performed, and a number of parameters, such as ejection fraction (EF), vital capacity (VC), 1 sec forced expiratory volume (FEV1.0), hemoglobin (Hg), C-reactive protein (CRP) levels were measured. We also calculated the prognostic nutritional index (PNI) and body mass index (BMI) and measured prealbumin (PreAlb), transferrin (Tf), and retinol binding protein (RBP) levels as well as CRP levels from postoperative days 1-30. The PNI is calculated using the following formula: $10 \times$ serum albumin $(\mathrm{g} / \mathrm{dL})+0.005 \times$ total lymphocyte count (per $\mathrm{mm}^{3}$ ).

\section{Statistical analysis}

Statistical analyses were performed using JMP version 12 software (SAS Institute Inc., Cary, NC, USA). The significance of differences between values in two different groups was assessed by the Mann - Whitney $U$-test. In a univariate analysis, a comparison of categorical variables was performed using the chi-squared test. Continuous data are expressed as the mean \pm standard deviation (SD). A receiver operating characteristic (ROC) curve was created to assess the ability of E-PASS and APACHE II scores to predict the incidence of complications. A ROC curve was generated and sensitivity was plotted against specificity. The area under the ROC curve (AUC) was used to evaluate discriminatory ability to detect postoperative complications. A cut-off value corresponding to maximum sensitivity and specificity was obtained using Youden's index from the ROC curve. The relationships between different continuous variables were quantified by Pearson's correlation by rank. A $p$-value of less than 0.05 was considered significant.

\section{Results}

Postoperative complications were developed in seven out of 30 patients, who were subsequently categorized into the complication group (Table 2). Among these patients, one had methicillin-resistant Staphylococcus aureus (MRSA) pneumonia, five had 
Table 1 Estimation of Physiologic Ability and Surgical Stress (E-PASS) and Acute Physiology and Chronic Health Evaluation (APACHE) II scoring systems

The E-PASS score consists of three parts for estimation of physiologic ability (PRS), surgical stress (SSS), and their comprehensive score(CRS). The formula for each score was as follows:

$P R S=-0.0686+0.00345 \times 1+0.323 \times 2+0.205 \times 3+0.153 \times 4+0.148 \times 5+0.0666 \times 6$

XI: age

X2: absence (0) or presence (1) of severe heart disease

X3: absence (0) or presence (I) of severe pulmonary disease

X4: absence (0) or presence (I) of diabetes mellitus

X5: performance status index (0-4)

X6: American Society of Anesthesiologists physiological status classification (1-5)

SSS $=-0.342+0.0139 \times 1+0.0392 \times 2+0.352 \times 3$

XI: blood loss/body weight $(\mathrm{g} / \mathrm{kg})$

X2: Operative time (hours)

X3: Extent of the skin incision (0: minor incision, 1: laparotomy or thoracotomy alone, 2: both laparotomy and thoracotomy)

CRS $=-0.328+0.396$ (PRS) +0.976 (SSS)

The APACHE II score is the sum of the acute physiology score (vital signs, oxygenation, laboratory values), the Glasgow coma score, age, and Choronic health points. The worst values during the first $24 \mathrm{~h}$ in the ICU should be used. Glasgow coma score(GCS) = eye-openig score + veabal score (intubated or nonintubated) score + motor score. For CCS component of acute physiology score, subtract GCS from 15 to obtain points assigned.

Acute Physiology Score

\begin{tabular}{|c|c|c|c|c|c|c|c|c|c|}
\hline Score & 4 & 3 & 2 & 1 & 0 & 1 & 2 & 3 & 4 \\
\hline Rectal temperature $\left({ }^{\circ} \mathrm{C}\right)$ & $\geq 41$ & $\begin{array}{l}39.0 \sim \\
40.9\end{array}$ & & $\begin{array}{l}38.5 \sim \\
38.9\end{array}$ & $\begin{array}{l}36.0 \sim \\
38.4\end{array}$ & $\begin{array}{l}34.0 \sim \\
35.9\end{array}$ & $\begin{array}{l}32.0 \sim \\
33.9\end{array}$ & $\begin{array}{l}30.0 \sim \\
31.9\end{array}$ & $\leq 29.9$ \\
\hline $\begin{array}{l}\text { Mean blood pressure } \\
(\mathrm{mmHg})\end{array}$ & $\geq 160$ & $\begin{array}{l}130 \sim \\
159\end{array}$ & $\begin{array}{l}110 \sim \\
129\end{array}$ & & $\begin{array}{l}70 \sim \\
109\end{array}$ & & $\begin{array}{l}50 \sim \\
69\end{array}$ & & $\leq 49$ \\
\hline Heart rate (beat/min) & $\geq 180$ & $\begin{array}{l}140 \sim \\
179\end{array}$ & $\begin{array}{l}110 \sim \\
139\end{array}$ & & & & $\begin{array}{l}55 \sim \\
69\end{array}$ & $\begin{array}{l}40 \sim \\
54\end{array}$ & $\leq 39$ \\
\hline Respiratory rate (breaths/min) & $\geq 50$ & $\begin{array}{l}35 \sim \\
49\end{array}$ & & $\begin{array}{l}25 \sim \\
34\end{array}$ & $\begin{array}{l}12 \sim \\
24\end{array}$ & $\begin{array}{l}10 \sim \\
11\end{array}$ & $\begin{array}{l}6 \sim \\
9\end{array}$ & & $\leq 5$ \\
\hline Arterial pH & $\geq 7.70$ & $\begin{array}{l}7.60 \sim \\
7.69\end{array}$ & & $\begin{array}{l}7.50 \sim \\
7.59\end{array}$ & $\begin{array}{l}7.33 \sim \\
7.49\end{array}$ & & $\begin{array}{l}7.25 \sim \\
7.32\end{array}$ & $\begin{array}{l}7.15 \sim \\
7.24\end{array}$ & $<7.15$ \\
\hline \multicolumn{10}{|l|}{$\begin{array}{l}\text { Oxygenation: A-aD02 orPa02 } \\
\text { (mmHg) }\end{array}$} \\
\hline $\begin{array}{l}\text { a. } \mathrm{FiO} 2>0.5 \text { record } \\
\text { A-aD02 }\end{array}$ & $\geq 500$ & $\begin{array}{l}350 \sim \\
499\end{array}$ & $\begin{array}{l}200 \sim \\
349\end{array}$ & & $<200$ & & & & \\
\hline b. Fi02 $\leq 0.5$ record $\mathrm{PaO} 2$ & & & & & $>70$ & $\begin{array}{l}61 \sim \\
70\end{array}$ & & $\begin{array}{l}55 \sim \\
60\end{array}$ & $<55$ \\
\hline Serum sodium (mmol/L) & $\geq 180$ & $\begin{array}{l}160 \sim \\
179\end{array}$ & $\begin{array}{l}155 \sim \\
159\end{array}$ & $\begin{array}{l}150 \sim \\
154\end{array}$ & $\begin{array}{l}130 \sim \\
149\end{array}$ & & $\begin{array}{l}120 \sim \\
129\end{array}$ & $\begin{array}{l}111 \sim \\
119\end{array}$ & $\leq 110$ \\
\hline Serum potassium(mmol/L) & $\geq 7.0$ & $\begin{array}{l}6.0 \sim \\
6.9\end{array}$ & & $\begin{array}{l}5.5 \sim \\
5.9\end{array}$ & $\begin{array}{l}3.5 \sim \\
5.4\end{array}$ & $\begin{array}{l}3.0 \sim \\
3.4\end{array}$ & $\begin{array}{l}2.5 \sim \\
2.9\end{array}$ & & $<2.5$ \\
\hline Serum creatinine) mg/dl) & $\geq 3.5$ & $\begin{array}{l}2.0 \sim \\
3.4\end{array}$ & $\begin{array}{l}1.5 \sim \\
1.9\end{array}$ & & $\begin{array}{l}0.6 \sim \\
1.4\end{array}$ & & $<0.6$ & & \\
\hline Hematocrit (\%) & $\geq 60$ & & $\begin{array}{l}50 \sim \\
59.9\end{array}$ & $46 \sim 49.9$ & $\begin{array}{l}30 \sim \\
45.9\end{array}$ & & $\begin{array}{l}20 \sim \\
29.9\end{array}$ & & $<20$ \\
\hline $\begin{array}{l}\text { White blood cell count } \\
(\times 1000)\end{array}$ & $\geq 40$ & & $\begin{array}{l}20 \sim \\
39.9\end{array}$ & $\begin{array}{l}15 \sim \\
19.9\end{array}$ & $\begin{array}{l}3 \sim \\
14.9\end{array}$ & & $\begin{array}{l}1 \sim \\
2.9\end{array}$ & & $<1$ \\
\hline \multicolumn{10}{|l|}{ Glasgow Coma Score } \\
\hline Eye Opening & \multicolumn{3}{|c|}{ verbal (Non-intubated) } & \multicolumn{3}{|c|}{ veabal (intubated) } & \multicolumn{3}{|c|}{ Motor Activity } \\
\hline 4-Spontaneous & \multicolumn{3}{|c|}{ 5-Oriented and talks } & \multirow{2}{*}{\multicolumn{3}{|c|}{ 5-Seemsable to talk }} & \multicolumn{3}{|c|}{ 6-Verbal command } \\
\hline 3-Verbal stimuli & \multicolumn{3}{|c|}{ 4-Disoricnted and talks } & & & & \multicolumn{3}{|c|}{ 5-Localizedto pain } \\
\hline
\end{tabular}


Table 1 Estimation of Physiologic Ability and Surgical Stress (E-PASS) and Acute Physiology and Chronic Health Evaluation (APACHE) II scoring systems (Continued)

\begin{tabular}{|c|c|c|c|}
\hline & & $\begin{array}{l}\text { 3-Questionableabilhy } \\
\text { to talk }\end{array}$ & \\
\hline 2-Painful stimuli & 3-Inappropriate words & 1-Generally unresponsive & 4-Withdraws from pain \\
\hline \multirow[t]{3}{*}{ 1-No response } & $\begin{array}{l}\text { 2-Incomprehensihle } \\
\text { sounds }\end{array}$ & & 3-Decorticate \\
\hline & 1-No response & & 2-Decerebrate \\
\hline & & & 1-No response \\
\hline
\end{tabular}

Points Assigned to Age and Chronic Disease

$\begin{array}{ll}\text { Age, Years } & \text { Score } \\ <45 & 0 \\ 45 \sim 54 & 2 \\ 55 \sim 64 & 3 \\ 65 \sim 74 & 5 \\ \geq 75 & 6\end{array}$

Chronic Health (History of Chronic Conditions) Score

None 0

if the patient is admitted after elective surgery 2

if the patient is admitted after emergency surgery or for a reason other than after 5 elective surgery

Abbreviations: A-aD02 alveolar-arterial oxygen difference, FiO2 fraction of inspired oxygen, $\mathrm{PaO2}$ partial pressure of oxygen

postoperative pulmonary disease, and 1 had MRSA bacteremia; there was no postoperative deaths. No significant differences were observed in age $(70.2 \pm 10.1$ years versus 63.5 \pm 13.5 years, $p=0.123)$, gender $(p=0.896)$, smoking $(p=0.746)$ or BMI $\left(21.3 \pm 3.8 \mathrm{~kg} / \mathrm{m}^{2}\right.$ versus $\left.21.6 \pm 2.6 \mathrm{~kg} / \mathrm{m}^{2}, p=0.341\right)$. Among the preoperative cardiac and respiratory parameters measured, no significant differences were observed in EF $(62.8 \pm 15.5 \%$ versus $69.5 \pm 5.5 \%, p=0.149), \mathrm{VC}(2.9 \pm 0.9 \mathrm{~L}$ versus $3.2 \pm 0.8 \mathrm{~L}, p=0.193), \mathrm{FEV} 1.0(2.0 \pm 0.6 \mathrm{~L}$ versus $2.3 \pm 0.7 \mathrm{~L}, p=0.150), \mathrm{Hg}(13.0 \pm 2.3 \mathrm{~g} / \mathrm{dL}$ versus $12.9 \pm 1.7 \mathrm{~g} / \mathrm{dL}, p=0.404)$, or CRP $(0.6 \pm 1.0 \mathrm{mg} / \mathrm{dL}$ versus $0.4 \pm 0.7 \mathrm{~g} / \mathrm{dL}, p=0.414)$ between patients with/without postoperative complications. Similarly, for the preoperative nutrition status, no significant differences were noted in PNI (46.6 \pm 5.8 versus $47.3 \pm 6.7, p=0.5)$, PreAlb (22.1 \pm $3.2 \mathrm{mg} / \mathrm{dL}$ versus $22.5 \pm 4.6 \mathrm{mg} / \mathrm{dL}, p=0.435)$, Tf $(253.7 \pm 54.7 \mathrm{mg} / \mathrm{dL}$ versus $226.6 \pm$ $27.7 \mathrm{mg} / \mathrm{dL}, p=0.136)$, or RBP $(2.85 \pm 0.5 \mathrm{mg} / \mathrm{dL}$ versus $2.90 \pm 0.9 \mathrm{mg} / \mathrm{dL}, p=0.318)$ between patients with/without postoperative complications. Regarding intraoperative results, the operative time and amount of blood lost during surgery, or length of hospital stay were similar between the two groups (operative time; $14.2 \pm 4.7 \mathrm{~h}$ versus $13.5 \pm 2.7 \mathrm{~h}$, $p=0.490$, blood loss; $476.7 \pm 336 \mathrm{mg}$ versus $405.7 \pm 242 \mathrm{mg} / \mathrm{dL}, p=0.323$, length of hospital days; $65.0 \pm 36.9$ days versus $78.6 \pm 46.2$ days, $p=0.221$ ) (Table 2).

The CRS, PRS, and APACHE II scores were significantly higher in patients with postoperative complications than in those without ones (CRS; $1.13 \pm 0.24$ versus $0.89 \pm 0.15$, $p<0.01$, PRS; $0.45 \pm 0.26$ versus $0.26 \pm 0.11, p<0.05$, APACHE II; $13 \pm 2.58$ versus 9.39 $\pm 3.43, p<0.05)$, whereas SSS was similar between the two groups $(0.69 \pm 0.17$ versus $0.64 \pm 0.13, p=0.199$ ) (Fig. 1).

The number of postoperative days until CRP levels decreased to $<1.0 \mathrm{mg} / \mathrm{L}$ correlated with APACHE II scores $(r=0.43, p<0.05)$, but not the CRS $(r=0.32, p=0.087)$ (Fig. 2). 
Table 2 Preoperative laboratory data and operative findings between patients with/without postoperative complications

\begin{tabular}{|c|c|c|c|}
\hline & Complications $(+)(n=7)$ & Complications $(-)(n=23)$ & $p$-value \\
\hline Age & $70.2 \pm 10.1$ & $63.5 \pm 13.5$ & 0.123 \\
\hline Gender & & & 0.896 \\
\hline Men & 5 & 17 & \\
\hline Women & 2 & 6 & \\
\hline Smoking & 1 & 4 & 0.746 \\
\hline BMl & $21.3 \pm 3.8$ & $21.6 \pm 2.6$ & 0.341 \\
\hline $\mathrm{EF}(\%)$ & $62.8 \pm 15.5$ & $69.5 \pm 5.5$ & 0.149 \\
\hline VC (L) & $2.9 \pm 0.9$ & $3.2 \pm 0.8$ & 0.193 \\
\hline$\% \mathrm{VC}$ & $97.2 \pm 14.6$ & $98.8 \pm 26.0$ & 0.206 \\
\hline FEV1.0 (L) & $2.0 \pm 0.6$ & $2.3 \pm 0.7$ & 0.150 \\
\hline FEV1.0\% & $72.5 \pm 6.7$ & $75.9 \pm 6.4$ & 0.156 \\
\hline $\mathrm{Hg}(\mathrm{g} / \mathrm{dL})$ & $13.0 \pm 2.3$ & $12.9 \pm 1.7$ & 0.404 \\
\hline CRP $(\mathrm{mg} / \mathrm{dL})$ & $0.6 \pm 1.0$ & $0.4 \pm 0.7$ & 0.414 \\
\hline $\mathrm{PNI}$ & $46.6 \pm 5.8$ & $47.3 \pm 6.7$ & 0.500 \\
\hline PreAlb (mg/dL) & $22.1 \pm 3.2$ & $22.5 \pm 4.6$ & 0.435 \\
\hline $\mathrm{Tf}(\mathrm{mg} / \mathrm{dL})$ & $253.7 \pm 54.7$ & $226.6 \pm 27.7$ & 0.136 \\
\hline $\mathrm{RBP}(\mathrm{mg} / \mathrm{dL})$ & $2.85 \pm 0.5$ & $2.90 \pm 0.9$ & 0.318 \\
\hline \multicolumn{4}{|l|}{ Diagnosis } \\
\hline Malignant tumor & 7 & 22 & \\
\hline Benign tumor & 0 & 1 & \\
\hline \multicolumn{4}{|l|}{ Operative approach } \\
\hline Radical neck dissection & 6 & 15 & \\
\hline Supraomohyoid Neck Dissection & 1 & 5 & \\
\hline Upper Neck Dissection & & 1 & \\
\hline \multicolumn{4}{|l|}{ Reconstruction method } \\
\hline PMMC flap & 3 & 6 & \\
\hline Forearm flap & 3 & 6 & \\
\hline Latissimus dorsi flap & & 6 & \\
\hline DP flap & & 3 & \\
\hline Operative time (hr) & $14.2 \pm 4.7$ & $13.5 \pm 2.7$ & 0.490 \\
\hline Blood loss (mg) & $476.7 \pm 336.6$ & $405.7 \pm 242.4$ & 0.323 \\
\hline \multicolumn{4}{|l|}{ Postoperative complications } \\
\hline Pneumonia & 6 & & \\
\hline MRSA bacteremia & 1 & & \\
\hline Length of hospital stay (days) & $65.0 \pm 36.9$ & $78.6 \pm 46.2$ & 0.221 \\
\hline
\end{tabular}

$B M I$ body mass index, EF ejection fraction, $V C$ vital capacity, FEV1.0 One second forced expiratory volume, $P N I$ prognostic nutritional index, PreAlb Prealbumin, If transferrin, $R B P$ retinol binding protein, $P M M C$ pectoralis major myocutaneous, $D P$ Delto-pectoral, MRSA methicillin-resistance Staphylococcus aureus

The ROC curve analysis calculated cut-off values of 1.01 for the CRS and 10.00 for APACHE II. The sensitivities and specificities of the CRS and APACHE II were 0.857 and 0.739 , and 1.000 and 0.478 , respectively. The AUCs of each model for the detection of postoperative complications were as follows: the CRS: 0.814 and APACHE II: 0.795 (Fig. 3). 

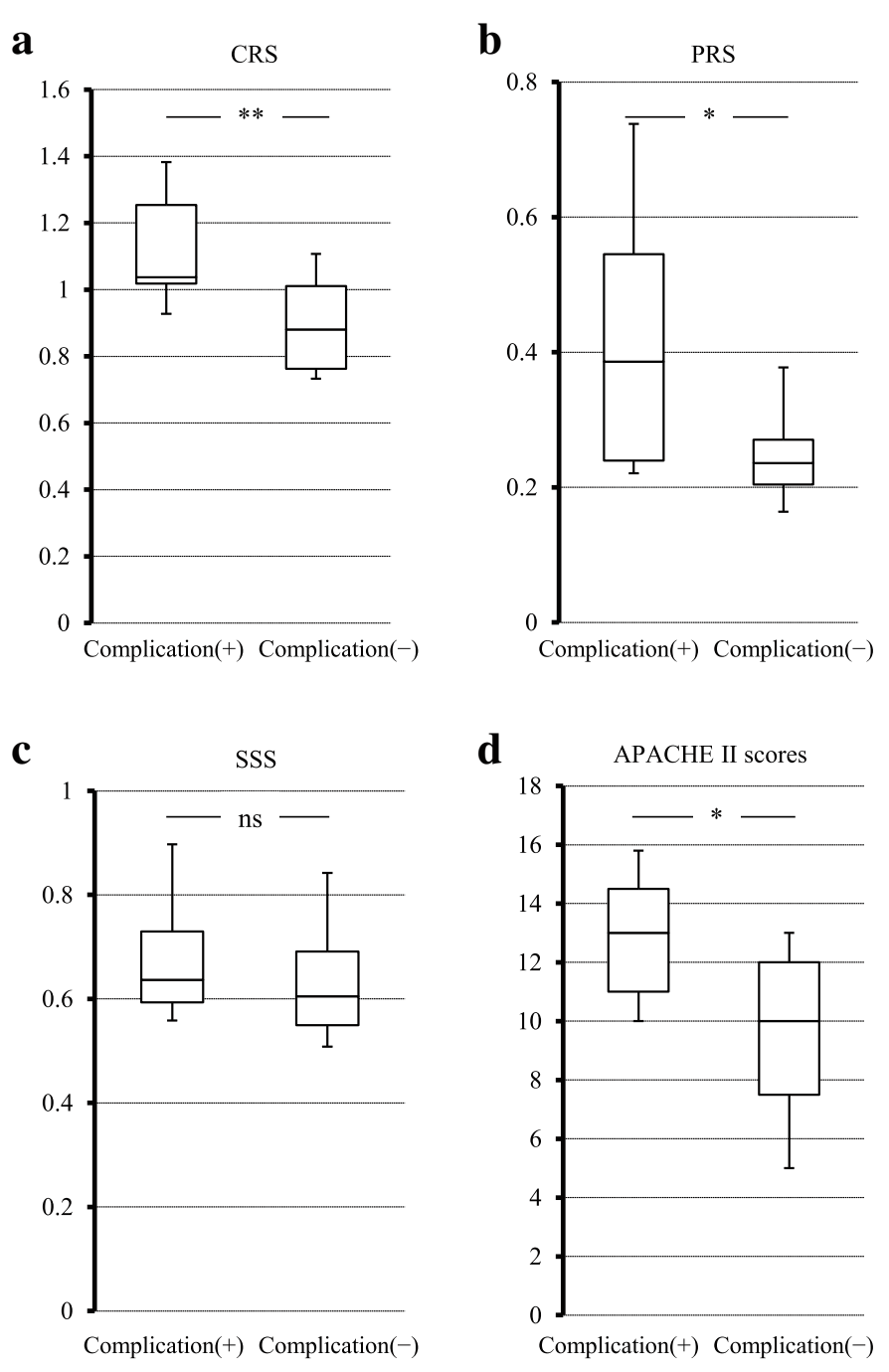

$$
\begin{aligned}
& * *: \mathrm{p}<0.01, *: \mathrm{p}<0.05 \\
& \text { Mann-Whitney U-test }
\end{aligned}
$$

Fig. 1 Comparison of E-PASS and APACHE II scores between patients with and without postoperative complications. Comparison of the comprehensive risk score (CRS): a, preoperative risk score (PRS): $\mathbf{b}$, surgical stress score (SSS): $\mathbf{c}$ and acute physiology and chronic health evaluation (APACHE) II score: $\mathbf{d}$ between patients with and without postoperative complications. CRS $(p<0.01)$, PRS $(p<0.05)$ and APACHE II $(p<0.05)$ scores were significantly higher in patients with than in those without postoperative complications. No significant difference was observed in SSS scores $(p=0.20)$ between the 2 groups

\section{Discussion}

In the present study, we evaluated the efficacy of the E-PASS and APACHE II scoring systems to predict postoperative complications in the field of oral and maxillofacial surgery. To the best of our knowledge, this is the first study to show the utility of the EPASS and APACHE II scoring systems for oral surgeons and, thus, may lead to the clinical use of these systems in pre- and postoperative management, surgical decisionmaking, and informed consent.

Postoperative complications following oral and maxillofacial surgery such as wound infections and swallowing and breathing difficulties have a significant impact on mobility, resulting in prolonged hospital stays and reduced quality of life among patients and, 
$\mathbf{a}$

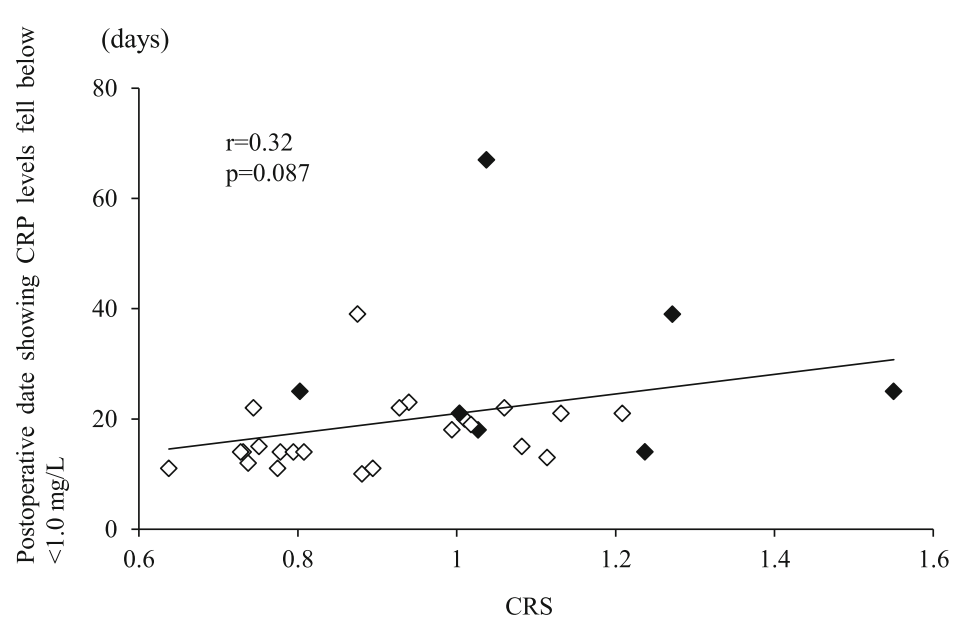

b

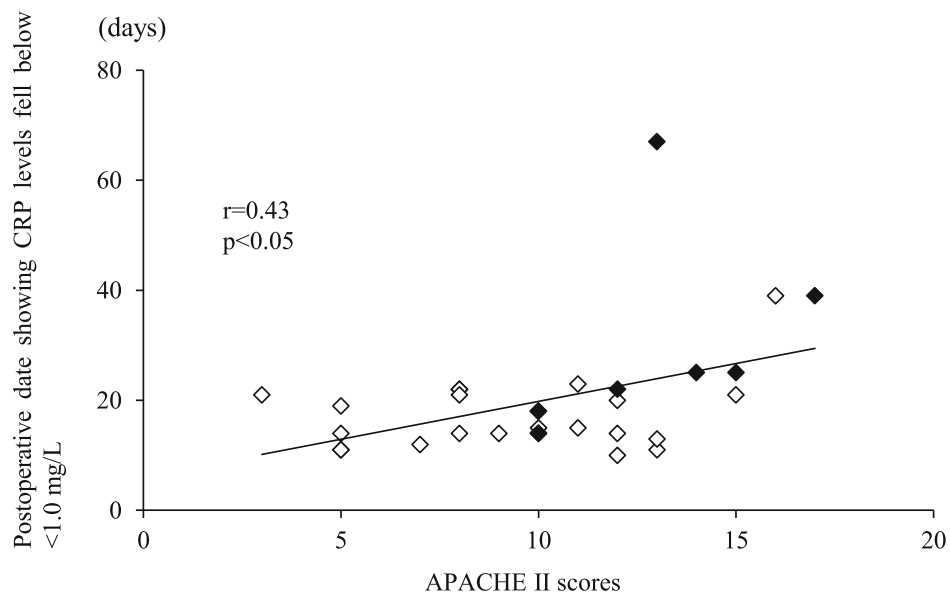

Fig. 2 Relationship between the number of postoperative days until C-reactive protein levels decreased to $<1.0 \mathrm{mg} / \mathrm{L}$ and the comprehensive risk score (CRS) (a) or acute physiology and chronic health evaluation (APACHE) II score: (b). Correlations were observed between APACHE II and the number of postoperative days until CRP levels decreased to $<1.0 \mathrm{mg} / \mathrm{L}(r=0.43, p<0.05)$. No correlations were found between CRS scores and the number of postoperative days until CRP levels decreased to $<1.0 \mathrm{mg} / \mathrm{L}(p=0.087)$. $\diamond$ : Without postoperative complications. $\bullet$ With postoperative complications

inevitably, increased health care costs [8]. In the present study, seven out of 30 patients were developed postoperative complications; six had pulmonary complications and one had bacteremia. Previous studies have shown that the incidence of postoperative pulmonary complications after surgery ranges between 3 and $48 \%[9,10]$. Major oral and maxillofacial surgeries with musculocutaneous flap reconstruction involve large skin incisions, thus postoperative pain and tension around the surgical site may contribute to several complications such as atelectasis, hypoventilation, and declined activity. Furthermore, the development of dysphagia in patients who undergo oral and maxillofacial surgery and its detrimental effects on functioning and the quality of life of these patients has been well documented [11]. Oral and maxillofacial lesions are heterogeneous because the anatomic sites of the lesions vary and many factors may influence swallowing process or respiration postoperatively in patients who have undergone oral and maxillofacial surgery. Therefore, surgery in the head and neck area is associated with a high risk of postoperative pulmonary complications 

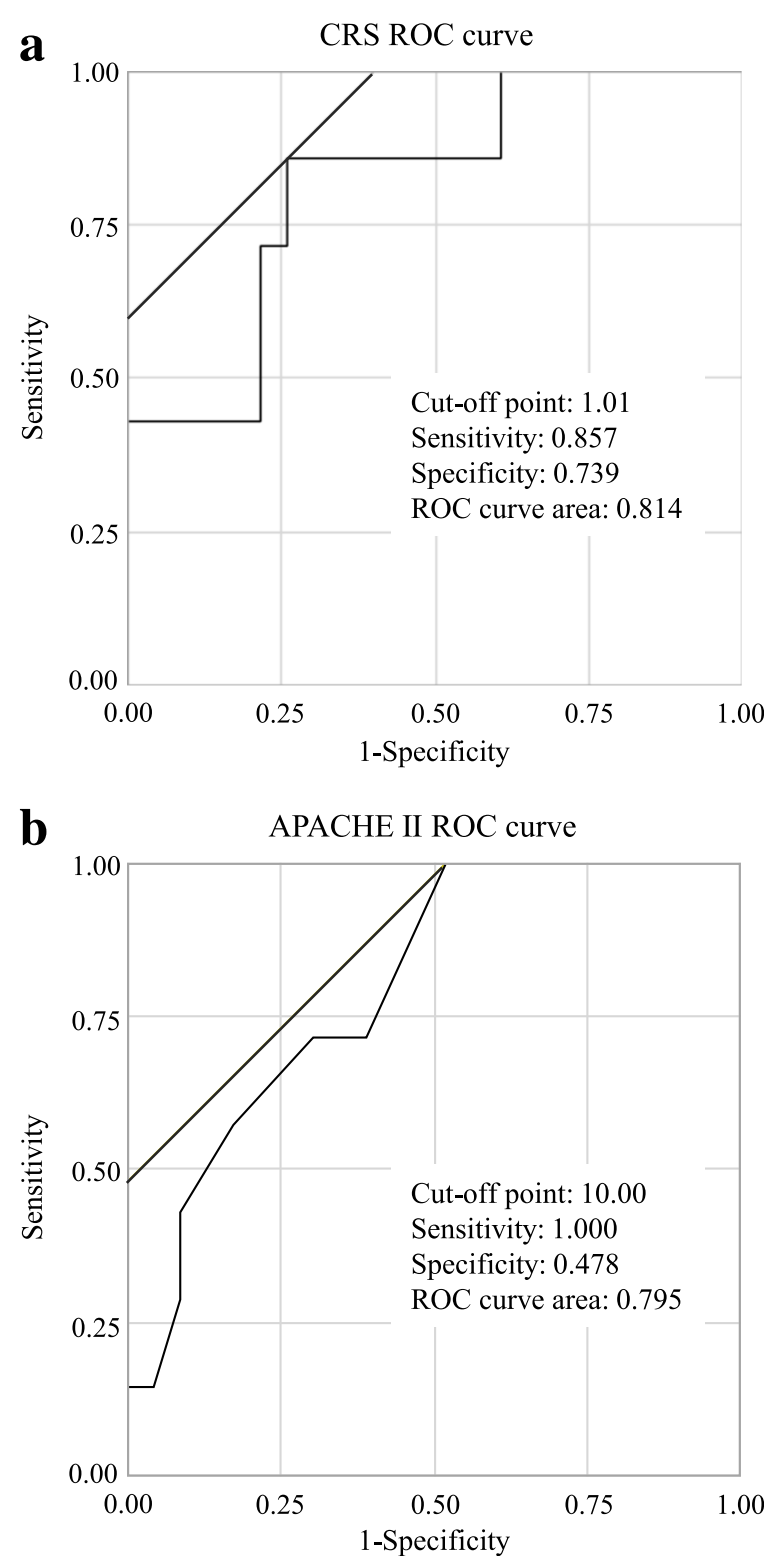

Fig. 3 Receiver operating characteristic (ROC) curve of the comprehensive risk score (CRS): (a) and acute physiology and chronic health evaluation (APACHE) II: (b) for predicting postoperative complications in oral and maxillofacial surgery. Approximate optimal cut-off points for predicting complications: CRS 1.01 (sensitivity: 85.7\%, specificity: 73.9\%, ROC curve area: 0.814) and APACHE I| 10.00 (sensitivity: 100.0\%, specificity: 47.8\%, ROC curve area: 0.795 )

$[10,12]$. In the present study, those with postoperative respiratory complications had various primary diseases, such as buccal, maxillary, and tongue carcinomas, and carcinoma of the floor of mouth. For the treatment of postoperative respiratory complications, tracheotomy and reintubation were needed in 3 and 1 of the 6 cases, respectively. There were no pulmonary comorbidity and preoperative respiratory function was within normal ranges. Hence, the development of analytical procedures that are sensitive to the factors influencing the relationship between postoperative pulmonary diseases and local states in the head and neck area after surgery are desired. 
Previous studies have shown that the most frequent complication in patients who undergo oral and maxillofacial surgery is postoperative infections [13]. Facial reconstruction procedures are immensely complex surgical procedures that are performed to replace tissue defects and restore anatomical structures as a result of various diseases, such as head and neck cancer and oral benign tumors. Therefore, an extensive surgical area is necessary for facial reconstruction and, inevitably, the incidence of postoperative infections is high. In the present study, one patient who underwent maxillary dissection for carcinoma of the maxilla and radical neck dissection developed MRSA pneumonia after 10 days postoperative. Another study revealed that postoperative infections are associated with cardiac disease and diabetes mellitus [14]; similarly, our patient had poor cardiac function (preoperative EF: 29.4\%) and diabetes mellitus.

Various scoring systems have been developed to predict postoperative morbidity and mortality. The ideal risk scoring tool has the following attributes: simple; easy to use; reproducible; accurate; reliable; objective; and available to all patients. Hattori and colleagues described the effectiveness of E-PASS scores to predict postoperative complications in colorectal patients [15]. However, only few studies have assessed this system in oral and maxillofacial surgery. We focused on patients undergoing oral and maxillofacial surgery with long operating times who were considered to have more severe surgical stress or a higher risk of postoperative complications than those undergoing minimally invasive surgery.

The CRS was calculated from the PRS (which includes perioperative patient condition factors) and SSS (including surgical condition factors) [2]. In the present study, the CRS was significantly higher in patients with postoperative complications than in those without ones (1.13 vs. $0.90 ; p<0.01$ ). Previous studies have suggested that patients with CRS $>1$ are at a particularly high risk of mortality while those with CRS $>0.5$ are at a high risk of morbidity [15]. Furthermore, the CRS, PRS, and SSS correlated with the duration of the hospital stay. These findings suggested that patients with a higher PRS are at a greater risk of perioperative morbidity, particularly in the field of vascular surgery, and the strong correlation between the PRS and outcomes may allow surgeons to predict risks in an individual patient before surgery [15]. In the present study, most patients had CRS > 1.0; only one patient with postoperative complications had a moderately high $\mathrm{CRS}$ score $(\mathrm{CRS}=0.81)$. Furthermore, PRS scores were significantly higher in patients with complications. We also used the number of postoperative days until CRP levels decreased to $<1.0 \mathrm{mg} / \mathrm{L}$ in order to investigate the improvement outcomes of patients, and the results obtained indicated that a relationship exists between PRS scores and outcomes. Regarding the accuracy of predicting postoperative complications, previous studies demonstrated that E-PASS accurately predicted postoperative mortality in the surgical treatment of hilar cholangiocarcinoma; the AUC to detect inhospital mortality was 0.842 for E-PASS [16]. Similar results were obtained in our study; we yielded an AUC value for the CRS to predict postoperative complications that was greater than 0.814 and calculated a cut-off value of 1.01, which was consistent with previous findings [16, 17]. Collectively, the results of the present study and previous findings support E-PASS models, particularly the CRS having a high predictive power in the field of oral and maxillofacial surgery. However, the operative type was not a factor associated with postoperative complications in our study; therefore, it remains unclear whether the type of surgery influences the validity of the scores obtained. 
Nevertheless, some independent factors for postoperative complications, such as operative type, anesthesia type, operative time, anesthesia time, blood loss, and intraoperative circulating volume are expected to contribute to the outcomes of surgery, and further considerations are needed in order to obtain insights into the relationship between intraoperative findings and outcomes.

The APACHE prognostic scoring system is a well-established validated tool for assessing the severity of disease and predicting hospital mortality using data obtained in the ICU admission [5, 18]. APACHE II provides a lot of information on factors influencing the prediction of outcomes, such as age, underlying diseases, and acute physiological conditions, which are crucial for severe morbidity and late mortality; therefore, this scoring system has been frequently used in many ICUs worldwide [6]. Previous studies indicated that the APACHE II scoring system accurately detects hospital mortality by evaluating an AUC of 0.84. Moreover, the best cut-off value in the ROC of this scoring system was $17[6,19]$.

.Therefore, we employed APACE II as an additional indicator to evaluate the clinical status of a patient and predict outcomes after long, highly invasive oral and maxillofacial surgery. As a result, we found that higher APACHE II scores correlated with an increased risk of postoperative complications, and was nearly identical to the E-PASS scoring system. Among our patients, APACHE II scores were significantly higher in patients with complications than in those without ones, a correlation was observed between APACHE II scores and the number of postoperative days until CRP levels decreased to $<1.0 \mathrm{mg} / \mathrm{L}$, and the ACU of APACHE II was 0.795. These results confirmed that APACHE II is a useful parameter that correlates with clinical changes in patients. Nevertheless, previous studies indicated that APACHE II $>17$ was associated with a high probability of postoperative morbidity and mortality [6]. In the present study, the median value of APACHE II in patients with complications was 13. A possible reason why our patients had lower APACHE II scores is few of them showed abnormalities in arterial $\mathrm{pH}$, renal functions, or serum electrolytes. Previous studies reported that the predictive accuracy of APACHE system was limited for mortality [19]; accordingly, models that combine additional baseline characteristics or other scoring systems may better assess disease severity, thus, improve the estimated risk of mortality over that with APACHE II alone. Prediction models based on multivariate analyses typically use a logistic regression analysis due to the advantage of its simpler interpretation of the relationship between predictive factors and outcomes [20]. Another study built a prediction model combining APACHE II scores, and found that the new model was more accurate than APACHE II alone for predicting hospital mortality [18]. Difficulties are associated with combining E-PASS and APACHE II scores; however, these scoring models may be useful in the management of patients after surgery; in other words, we recommend the application of E-PASS scores to assess the pre- and intraoperative statuses of patients and APACHE II scores to determine postoperative severity.

The precision and accuracy of surgery are important for all surgeons, and elaborate patient management in the perioperative period is also required in order to prevent postoperative complications. Previous studies have indicated that postoperative prognoses after various surgeries are reflected by the preoperative nutritional conditions of patients [21]. Malnutrition has been identified as an independent risk factor for morbidity and mortality and is associated with a significantly longer hospital stay [22]. 
Consequently, a good nutritional status is important to avoid postoperative complications in patients undergoing oral and maxillofacial surgery and affects the body's defense mechanisms in a number of ways. Low levels of serum albumin, nutritional markers such as PNI, and preoperative BMI are major risk factors for adverse postoperative outcomes $[17,23]$. These factors have been widely adopted in a large number of facilities, including our own, in order to evaluate the nutritional status of patients. Furthermore, we have adopted more sensitive nutritional markers such as PreAlb, Tf, and RBP, the so-called rapid turnover protein in the past several years; these markers have a half-life in plasma of 2 to 7 days, which is markedly shorter than that of serum albumin [24]. Therefore, PreAlb, Tf, and RBP are more sensitive to changes in the proteinenergy status than albumin, and their concentrations closely reflect recent dietary intake rather than the overall nutritional status. In the present study, no significant differences were observed in the nutritional status of patients with/without postoperative complications, and the measured values of nutritional markers mostly remained within normal ranges. This may be attributed to an attempt to intervene in nutritional control in the early phase of the preoperative period with consideration for the importance of nutritional support to prevent postoperative complications. Nevertheless, we cannot exclude the possibility that the incidence of postoperative complications after oral and maxillofacial surgery and, similarly, about 240 million people undergo surgery worldwide, with postoperative complications occasionally reported. In the future, ideal predictors for postoperative complications will be established to assess perioperative conditions from a wider perspective.

A limitation of the present study was that it is a retrospective analysis, which restricts our investigation of data archived during the perioperative period and the number of patients available was small. Consequently, the sample size was insufficient to analyze some intervening variables such as gender, disease, tumor size, and location. Moreover, other factors than that those included in the E-PASS and APACHE II scoring systems could be relevant for predicting postoperative complications and hence, it is important to comprehensively evaluate the risk factor of postoperative complications.

\section{Conclusions}

In summary, we herein analyzed the predictive powers of the E-PASS and APACHE II scoring systems in patients undergoing oral maxillofacial surgery. Our results suggest that their predictive values are promising for oral surgeons. The E-PASS and APACHE II scoring systems will be useful for surgical decision-making, informed consent, and assessing the quality of care in this field. These efforts will improve the quality of surgical performance.

\footnotetext{
Abbreviations ROC: Receiver operating characteristic; SSS: Surgical stress score

Acknowledgements

This work was supported by JSPS KAKENHI Grant Number JP16K11692.

Funding

This study received no funding.
}

APACHE: Acute Physiology, Age, and Chronic Health Evaluation; CRP: C-reactive protein; CRS: Comprehensive risk score; E-PASS: Estimation of Physiologic Ability and Surgical Stress; ICU: Intensive Care Units; PRS: Preoperative risk score; 
Authors' contributions

$\mathrm{KI}, \mathrm{YK}, \mathrm{TY}, \mathrm{JM}, \mathrm{ST}, \mathrm{HH}, \mathrm{EN}$, and NN participated in drafting and revising the manuscript. All authors have read and approved the final manuscript.

Ethics approval and consent to participate

This retrospective study and clinical record reviews were approved by the Institutional Review Board of Kagoshima University (No. 28-101)

\section{Consent for publication}

Not applicable.

\section{Competing interests}

The authors declare that they have no competing interests.

\section{Publisher's Note}

Springer Nature remains neutral with regard to jurisdictional claims in published maps and institutional affiliations.

\section{Author details}

${ }^{1}$ Department of Oral and Maxillofacial Surgery, Field of Maxillofacial Rehabilitation, Kagoshima University Graduate School of Medical and Dental Sciences, 8-35-1 Sakuragaoka, Kagoshima 890-8544, Japan. ²Department of Emergency and Intensive Care Medicine, Faculty of Medicine, Kagoshima University, Kagoshima, Japan.

Received: 14 July 2017 Accepted: 22 February 2018

Published online: 05 April 2018

\section{References}

1. Cremer J, Martin M, Redl H, Bahrami S, Abraham C, Graeter T, Haverich A, Schlag G, Borst HG. Systemic inflammatory response syndrome after cardiac operations. Ann Thorac Surg. 1996;61:1714-20.

2. Haga Y, Ikei S, Ogawa M. Estimation of physiologic ability and surgical stress (E-PASS) as a new prediction scoring system for postoperative morbidity and mortality following elective gastrointestinal surgery. Surg Today. 1999;29: 219-25.

3. Ganly I, Patel S, Matsuo J, Singh B, Kraus D, Boyle J, Wong R, Lee N, Pfister DG, Shaha A, Shah J. Postoperative complications of salvage total laryngectomy. Cancer. 2005;103:2073-81.

4. Hattori H, Kamiya J, Shimada H, Akiyama H, Yasui A, Kuroiwa K, Oda K, Ando M, Kawamura T, Harada A, Kitagawa Y, Fukata S. Assessment of the risk of postoperative delirium in elderly patients using E-PASS and the NEECHAM confusion scale. Int J Geriat Psychiatry. 2009;24:1304-10.

5. Knaus WA, Zimmerman JE, Wagner DP, Draper EA, Lawrence DE. APACHE-acute physiology and chronic health evaluation: a physiologically based classification system. Crit Care Med. 1981;9(8):591-7.

6. Cho DY, Wang YC. Comparison of the APACHE III, APACHE II and Glasgow coma scale in acute head injury for prediction of mortality and functional outcome. Intensive Care Med. 1997;23:77-84.

7. Dindo D, Demartines N, Clavien P-A. Classification of surgical complications. Ann Surg. 2004;240:205-13.

8. Shigeishi H, Ohta K, Takechi M. Risk factors for postoperative complications following oral surgery. J Appl Oral Sci. 2015;23:419-23.

9. Song K, Rong Z, Yang X, Yao Y, Shen Y, Shi D, Xu Z, Chen D, Zheng M, Jiang Q. Early pulmonary complications following Total knee arthroplasty under general anesthesia: a prospective cohort study using CT scan. Biomed Res Int. 2016:2016:4062043

10. Loeffelbein DJ, Julinek A, Wolff K-D, Kochs E, Haller B, Haseneder R. Perioperative risk factors for postoperative pulmonary complications after major oral and maxillofacial surgery with microvascular reconstruction: a retrospective analysis of 648 cases. J Craniomaxillofac Surg. 2016;44:952-7.

11. Nund RL, Ward EC, Scarinci NA, Cartmill B, Kuipers P, Porceddu SV. Carers' experiences of dysphagia in people treated for head and neck cancer: a qualitative study. Dysphagia. 2014;29:450-8.

12. Huang Z-s, Chen W-I, Huang Z-q, Yang Z-h. Dysphagia in tongue cancer patients before and after surgery. J Oral Maxillofac Surg. 2016;74(10):1-6.

13. Prakasam S, Stein K, Lee MK, Rampa S, Nalliah R, Allareddy V, Allareddy V. Prevalence and predictors of complications following facial reconstruction procedures. Int J Oral Maxillofac Surg. 2016:45:735-42.

14. Tang TY, Walsh SR, Fanshawe TR, Seppi V, Sadat U, Hayes PD, Varty K, Gaunt ME, Boyle JR. Comparison of riskscoring methods in predicting the immediate outcome after elective open abdominal aortic aneurysm surgery. Eur J Vasc Endovasc Surg. 2007;34:505-13.

15. Tang T, Walsh SR, Fanshawe TR, Gillard JH, Sadat U, Varty K, Gaunt ME, Boyle JR. Estimation of physiologic ability and surgical stress (E-PASS) as a predictor of immediate outcome after elective abdominal aortic aneurysm surgery. Am J Surg. 2007;194:176-82.

16. Haga Y, Miyamoto A, Wada Y, Takami Y, Takeuchi H. Value of E-PASS models for predicting postoperative morbidity and mortality in resection of perihilar cholangiocarcinoma and gallbladder carcinoma. HPB. 2016;18: $271-8$.

17. Tominaga T, Takeshita H, Takagi K, Kunizaki M, To K, Abo T, Hidaka S, Nanashima A, Nagayasu T, Sawai T. E-PASS score as a useful predictor of postoperative complications and mortality after colorectal surgery in elderly patients. Int J Color Dis. 2015;31:217-25.

18. Li G, Thabane L, Cook DJ, Lopes RD, Marshall JC, Guyatt G, Holbrook A, Akhtar-Danesh N, Fowler RA, Adhikari NKJ, Taylor R, Arabi YM, Chittock D, Dodek P, Freitag AP, Walter SD, Heels-Ansdell D, Levine MAH. Risk factors for and 
predictionof mortality in critically ill medical-surgical patients receiving heparin thromboprophylaxis. Ann Intensive Care. 2016;6(1):1-12.

19. Siontis GCM. Predicting death. Arch Intern Med. 2011;171:1721.

20. Adams ST, Leveson SH. Clinical prediction rules. BMJ. 2012;344:d8312.

21. Nozoe T, Kohno M, Iguchi T, Mori E, Maeda T, Matsukuma A, Ezaki T. The prognostic nutritional index can be a prognostic indicator in colorectal carcinoma. Surg Today. 2011;42:532-5.

22. Thomas MN, Kufeldt J, Kisser U, Hornung H-M, Hoffmann J, Andraschko M, Werner J, Rittler P. Effects of malnutrition on complication rates, length of hospital stay, and revenue in elective surgical patients in the G-DRGsystem. Nutrition. 2016;32:249-54.

23. Guo C-B, Ma D-Q, Zhang K-H, Hu X-h. Relation between nutritional state and postoperative complications in patients with oral and maxillofacial malignancy. Br J Oral Maxillofac Surg. 2007:45:467-70.

24. Shenkin A. Serum prealbumin: is it a marker of nutritional status or of risk of malnutrition? Clin Chem. 2006;52 2177-9.

Submit your next manuscript to BioMed Central and we will help you at every step:

- We accept pre-submission inquiries

- Our selector tool helps you to find the most relevant journal

- We provide round the clock customer support

- Convenient online submission

- Thorough peer review

- Inclusion in PubMed and all major indexing services

- Maximum visibility for your research

Submit your manuscript at www.biomedcentral.com/submit 